\title{
Comparative Efficacy of Certain Bio-Pesticides against Tomato Fruit Borer, Helicoverpa armigera (Hub.)
}

\author{
Renu Choudhary ${ }^{1}$, Ashwani Kumar ${ }^{1}$, G.C. Jat ${ }^{2}$, Vikram $^{1}$ and H.L. Deshwal ${ }^{3}$ \\ ${ }^{1}$ Department of Entomology, Sam Higginbottom Institute of Agriculture, Technology and \\ Sciences, (Formerly Allahabad Agricultural Institute), (Deemed to-be-University), Allahabad - \\ U.P., (India) - 211007 \\ ${ }^{2}$ Department of Entomology, Rajasthan College of Agriculture, (MPUAT) Udaipur, Rajasthan \\ 313001, India \\ ${ }^{3}$ Department of Entomology, College of Agriculture and Agriculture Research Station, (SKRAU) \\ Bikaner, Rajasthan, India \\ *Corresponding author
}

\section{A B S T R A C T}

Keywords

Helicoverpa armigera (Hub.), HaNPV,

Spinosad, B:C Ratio and Tomato variety Selection 22.

\section{Article Info}

Accepted:

14 June 2017

Available Online:

10 August 2017
The field experiment on "Comparative efficacy of certain bio-pesticides against tomato fruit borer, Helicoverpa armigera (Hub.)" was conducted during 2014-15 at Central Research Farm and Department of Entomology SHIATS, Naini, Allahabad (U.P). The relative efficacy of biorational- insecticides viz., spinosad $45 \mathrm{SC}(0.005 \%)$, quinalphos $25 \mathrm{EC}(0.05 \%)$, HaNPV (350 LE/ha), Beauveria bassiana (0.3ml/lit), Verticillium lecanii $(2.5 \mathrm{~kg} / \mathrm{ha})$ and Metarhizium anisopliae $(1000 \mathrm{ml} / \mathrm{ha})$ were evaluated against fruit borer (Helicoverpa armigera). The data on incremental percent reduction of different treatments revealed that the treatment Spinosad (74.97\%), followed by quinalphos (66.31\%), HaNPV $(59.74 \%)>$ Beauveria bassiana $(57.58 \%)>$ Verticillium lecanii $(47.10 \%)>$ Metarhizium anisopliae (44.46\%) found to be the most economically viable treatment. The highest cost benefit ratio was obtained from quinalphos (1:15.68) and the application of biorational- insecticides two spray at 15 days interval during rabi 2014-15.

\section{Introduction}

Tomato (Lycopersicon esculentum Mill) is one of the most important vegetables in the world, ranking second in importance to potato in many countries. It is a warm season crop. It is grown as an off-season vegetable in the hills of India and farmers fetch good income after sending their produce in the plains from June to September. Tomato supplies vitamin $\mathrm{C}$ and adds variety of colors and flavors to the foods. The fruits are eaten raw or cooked.
Large quantities of tomatoes are used to prepare soup, juice, ketchup, puree, pickle, paste and powder (Choudhary, 2002). About 16 insect and other pest species which caused damage to tomato crop in India Bhutani, (1977). Among the various pests, the tomato fruit borer, Helicoverpa armigera (Hub.) (Lepidoptera: Noctuidae) is the most destructive. The crop losses by $H$. armigera the extent of 80 per cent have been reported 
by Singh and Singh, (1975) and Yadav, (1980). The insect has developed resistance against many recommended insecticides (Srinivasan and Krishnamoorthy, 1992). Therefore, eco-friendly approaches have been evaluated for the management of $H$. armigera in tomato (Sivaprakasam, 1996, Khanam et al., 2003 and Selvanarayanan and Narayanasamy, 2006). There are some encouraging reports on the use of bio-rational insecticides against tomato fruit borer (Helicoverpa armigera) by Tripathi and Singh, (2005) and Dhaka et al., (2010). Such studies on tomato will be more fruitful because the fruits are free from toxic residues of insecticides. The pest is highly polyphagous and is reported on nearly 181 host plants Manjunath et al., (1987). The efficacy of different sequential application of nucleopolyhedro virus of $H$. armigera (HaNPV), Bacillus thuringiensis var. kurstaki Berliner (Bt. K.), neemazol and spinosad as the alternatives to the synthetic chemical pesticides for the sustainable management of Helicoverpa armigera on tomato ( Ravi et al., 2008).

\section{Materials and Methods}

\section{Layout and design}

The experiment on "Comparative efficacy of certain bio-pesticides against tomato fruit borer, Helicoverpa armigera (Hub.)" in trans Yamuna region of Allahabad. In Allahabad region at field condition was carried out during October 2014 to April, 2015 at
Agricultural Research Farm at SHIATS (Allahabad Agricultural Institute) Deemed University, Allahabad, Uttar Pradesh, India. The experiment was conducted in the randomized block design (RBD) with seven treatments schedules including control, and each treatment schedule was replicated two time at 15 days interval initiating first spray in the last week of February when the pest infestation started viz., 28 February and 14 March during rabi 2015, respectively. The tomato variety Selection -22 was transplanted on 27 January during rabi 2015. The plot size was $3.60 \times 3.60 \mathrm{~m}^{2}$ with row to row and plant to plant spacing of $45 \times 45 \mathrm{~cm}$, respectively.

The details of different treatment schedules are as follows

\section{Management schedule of treatments}

Pre-calibrated knap sack sprayer was used for spraying the biopesticides care was taken to check the drift of insecticides, by putting polythene sheet screen around each plot at the time of spraying. In all sprays were applied, first spray was done during the last week of February and subsequent second spray was applied at 15 days interval.

\section{Observations}

Pretreatment population of Helicoverpa armigera (Hub.) was recorded 24 hours before the scheduled spray.

Layout of Experimental Field

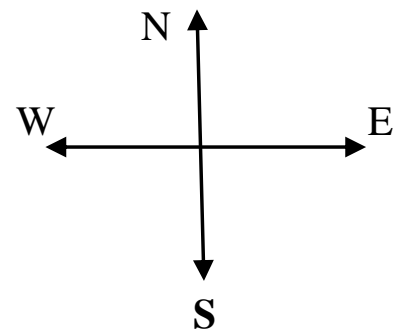




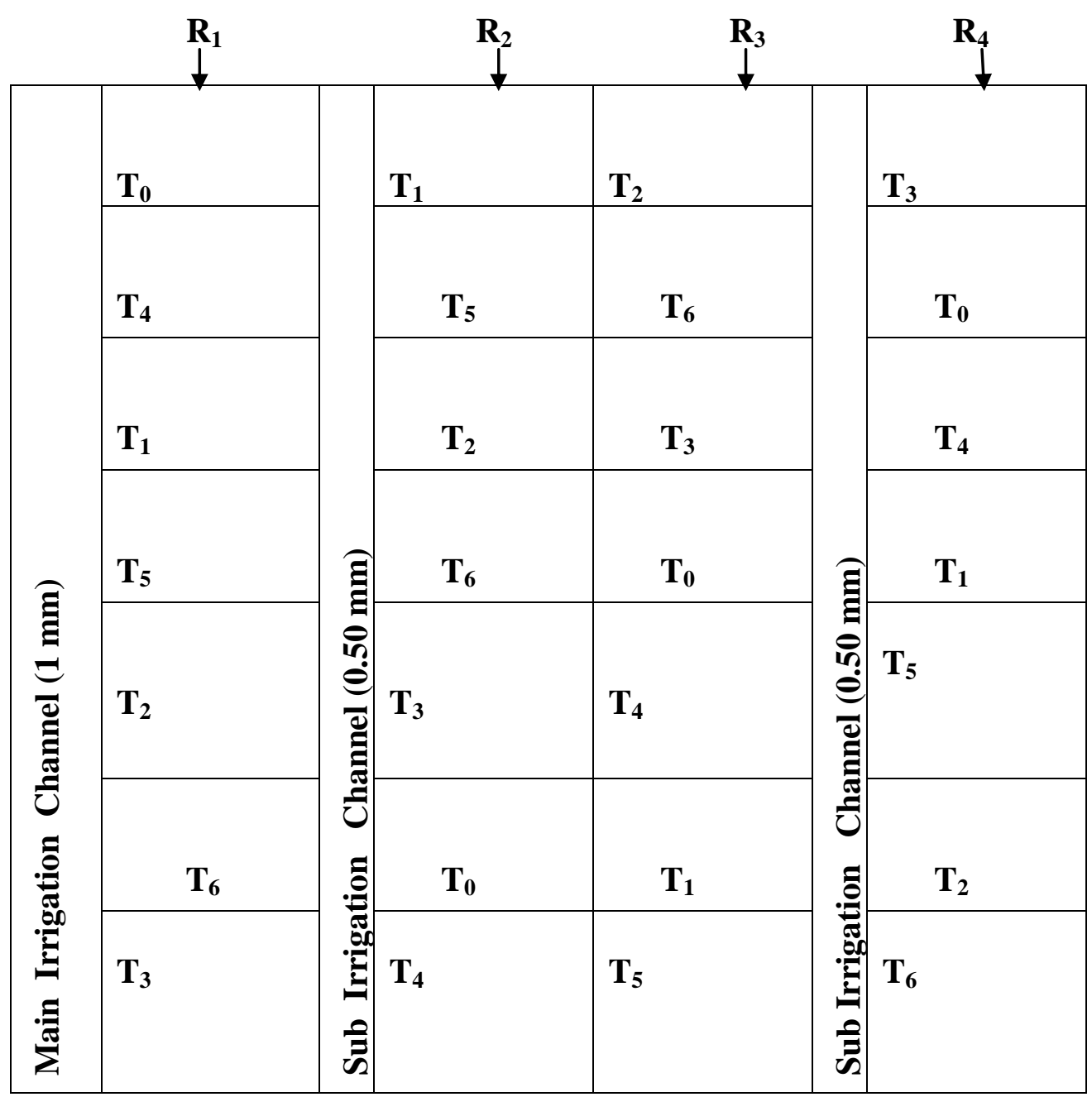

Table.1 Details of treatments

\begin{tabular}{|c|c|c|c|}
\hline Treatment & Chemical name & Dose (concentration) & References \\
\hline $\mathbf{T}_{\mathbf{0}}$ & Control & -------- & ---------- \\
\hline $\mathbf{T}_{1}$ & Verticillium lecanii & $2.5 \mathrm{~kg} / \mathrm{ha}$ & Kaur and Singh (2013) \\
\hline $\mathbf{T}_{2}$ & Metarhizium anisopliae & $1000 \mathrm{ml} / \mathrm{ha}$ & Lad et al., (2009) \\
\hline $\mathbf{T}_{3}$ & Beauveria bassiana & $0.3 \mathrm{ml} /$ litre & $\begin{array}{l}\text { Sreekanth and } \\
\text { Seshamahalakshmi (2010) }\end{array}$ \\
\hline $\mathbf{T}_{4}$ & HaNPV & $250 \mathrm{LE} / \mathrm{ha}$ & Kaur and Singh (2013) \\
\hline \multirow[t]{2}{*}{$\mathbf{T}_{5}$} & $\begin{array}{l}\text { Quinalphos } \\
\text { (Organophosphate) }\end{array}$ & $0.05 \%$ (2ml/lit.) & Naik et al., (2013) \\
\hline & Spinosad (Spinosyns) & $0.005 \%$ (0.1 ml/lit.) & Shinde et al., (2011) \\
\hline
\end{tabular}


Post treatment population of $H$. armigera was recorded on 3, 7 and 10 day after each spray, on 5 plants were selected randomly in each plot and tagged from each plot.

\section{Preparation of insecticidal solution}

The insecticidal spray of desired concentration as per treatment was freshly prepared every time at the time of experimentation just before the start of spraying operations. The spray solution of a desired concentration was prepared by adopting the following formula (Katyayan, 2010).

$V=\frac{C x A}{\text { a.i. } \%}$

Where,

$\mathrm{V}=$ Volume/ weight of commercial insecticide $\mathrm{ml}$ or $\mathrm{gm}$.

$\mathrm{C}=$ Concentration required.

$\mathrm{A}=$ Volume of solution to be prepared.

a.i. $(\%)=$ Percentage active ingredient

\section{Application of spray solution}

Spray solution was applied with the help of hand compression sprayer. Spraying was done at dawn and desk time and their must not be much wind currents.

\section{Reduction per cent by fruit borer}

The total numbers of infested and un infested plants at fruiting stage were counted from selected five plants of each plot. Thus the larvae was calculated using the following formula:

\begin{tabular}{|c|c|c|c|c|c|c|}
\hline $\begin{array}{l}\mathbf{T}_{4} \\
72.22\end{array}$ & $\begin{array}{r}T_{5} \\
63.0\end{array}$ & $\begin{array}{c}\mathbf{T}_{6} \\
56.07\end{array}$ & $\begin{array}{l}\mathbf{T}_{1} \\
\mathbf{5 5 . 4 2}\end{array}$ & $\begin{array}{c}\mathbf{T}_{2} \\
\mathbf{4 4 . 3 7}\end{array}$ & $\begin{array}{c}\mathbf{T}_{3} \\
\mathbf{4 3 . 4 2}\end{array}$ & $\begin{array}{r}\mathbf{T}_{\mathbf{0}} \\
\mathbf{0 . 0 0}\end{array}$ \\
\hline
\end{tabular}

Reduction percent $=$

Control--Treatment

Control x 100

\section{Benefit cost ratio}

Gross return was calculated by multiplying total yield with the market price of the produce. Cost of cultivation and cost of treatment imposition was deducted from the gross returns, to find out net returns and cost benefit ratio by following formula

$\mathrm{B}: \mathrm{C}=$

Gross Returns

Total Cost of Incurred

Where B: C = Benefit Cost Ratio

\section{Results and Discussion}

The results of studies undertaken during Rabi, 2014-15 on "Comparative efficacy of certain bio-pesticides against tomato fruit borer, Helicoverpa armigera (Hubner)] in trans yamuna region of Allahabad" are presented in the following heads.

\section{First spray}

\section{Reduction of fruit borer (First spray)}

The data on per cent reduction of fruit borer before spraying (Table 2) revealed that the results were statistically non significant. $3^{\text {rd }}$ day after $1^{\text {st }}$ spraying. The descending order of different treatment is given below. Some treatments were non significantly and some significantly superior over control. 
The data on population reduction percent of fruit borer, (Helicoverpa armigera) on $3^{\text {rd }}$ day after spraying showed that all the treatment was significantly superior over control. Maximum reduction was observed with spinosad $(72.22 \%)$, followed by quinalphos (63.03\%), HaNPV (56.07\%), Beauveria bassiana (55.42\%) Verticillium lecanii (44.37\%), and Metarhizium anisopliae (44.37\%), were most effective treatment.

Table.2 Comparative efficacy of certain bio-pesticides against tomato fruit borer [Helicoverpa armigera (Hubner)] on different days after 1st spray during Rabi season

2014-2015

\section{\% reduction in Helicoverpa armigera}

\begin{tabular}{|c|c|c|c|c|c|c|}
\hline & Treatment & Before & 3DAS & 7DAS & 14DAS & Mean \\
\hline $\mathrm{T}_{1}$ & Verticillium lecanii & 4.95 & $\begin{array}{c}55.42 \\
(\mathbf{4 8 . 1 1})^{*}\end{array}$ & $\begin{array}{c}58.95 \\
(\mathbf{5 0 . 1 5})^{*}\end{array}$ & $\begin{array}{c}46.27 \\
(\mathbf{4 2 . 8 6})^{*}\end{array}$ & 53.54 \\
\hline $\mathrm{T}_{2}$ & Beauveria bassiana & 5.4 & $\begin{array}{c}44.37 \\
(\mathbf{4 1 . 7 6})^{*}\end{array}$ & $\begin{array}{c}45.55 \\
(\mathbf{4 2 . 4 4})^{*}\end{array}$ & $\begin{array}{c}44.85 \\
(\mathbf{4 2 . 0 4})^{*}\end{array}$ & 44.92 \\
\hline $\mathrm{T}_{3}$ & Metarhizium anisopliae & 4.8 & $\begin{array}{c}43.42 \\
(\mathbf{4 1 . 2 2})^{*}\end{array}$ & $\begin{array}{c}44.56 \\
(\mathbf{4 1 . 8 7})^{*}\end{array}$ & $\begin{array}{c}35.17 \\
(\mathbf{3 6 . 3 7})^{*}\end{array}$ & 41.05 \\
\hline $\mathrm{T}_{4}$ & Spinosad & 5.05 & $\begin{array}{c}72.22 \\
(\mathbf{5 8 . 2 0})^{*}\end{array}$ & $\begin{array}{c}73.85 \\
(\mathbf{5 9 . 2 5})^{*}\end{array}$ & $\begin{array}{c}70.77 \\
(\mathbf{5 7 . 2 7})^{*}\end{array}$ & 72.28 \\
\hline $\mathrm{T}_{5}$ & Quinalphos & 5.05 & $\begin{array}{c}63.07 \\
(\mathbf{5 2 . 5 8})^{*}\end{array}$ & $\begin{array}{c}65.50 \\
(\mathbf{5 4 . 0 3})^{*}\end{array}$ & $\begin{array}{c}63.03 \\
(\mathbf{5 2 . 5 6})^{*}\end{array}$ & 63.86 \\
\hline $\mathrm{T}_{6}$ & HaNPV & 4.7 & $\begin{array}{c}56.07 \\
(\mathbf{4 8 . 4 9})^{*}\end{array}$ & $\begin{array}{c}59.52 \\
(\mathbf{5 0 . 4 9})^{*}\end{array}$ & $\begin{array}{c}54.30 \\
(\mathbf{4 7 . 4 6})^{*}\end{array}$ & 56.63 \\
\hline $\mathrm{T}_{0}$ & $\begin{array}{l}\text { Untreated control } \\
\text { Water spray }\end{array}$ & 4.95 & $\begin{array}{c}0.00 \\
(\mathbf{0 . 5 4})^{*}\end{array}$ & $\begin{array}{c}0.00 \\
(\mathbf{0 . 5 4})^{*}\end{array}$ & $\begin{array}{c}0.00 \\
(\mathbf{0 . 5 4})^{*}\end{array}$ & 0.0 \\
\hline & Over all Mean & & 47.79 & 49.70 & 44.91 & \\
\hline & F- test & & $S$ & $S$ & $S$ & \\
\hline & S. Ed. $( \pm)$ & & 0.96 & 0.83 & 0.89 & \\
\hline & C. D. $(P=0.05)$ & & & & & \\
\hline & & & 2.03 & 1.76 & 1.87 & 4.60 \\
\hline
\end{tabular}


Table.3 Comparative efficacy of certain bio-pesticides against tomato fruit borer [Helicoverpa armigera (Hubner)] on different days after 2nd spray during Rabi season 2014-2015

\begin{tabular}{|c|c|c|c|c|c|c|}
\hline & & \multicolumn{5}{|c|}{ \% reduction in Helicoverpa armigera } \\
\hline & Treatment & Before & 3DAS & 7DAS & 14DAS & Mean \\
\hline $\mathrm{T}_{1}$ & Verticillium lecanii & 2.6 & $\begin{array}{c}57.95 \\
(\mathbf{4 9 . 5 7 )}\end{array}$ & $\begin{array}{c}62.72 \\
(\mathbf{5 2 . 3 7})^{*}\end{array}$ & $\begin{array}{c}64.22 \\
(\mathbf{5 3 . 2 6})^{*}\end{array}$ & 61.63 \\
\hline $\mathrm{T}_{2}$ & Beauveria bassiana & 3.15 & $\begin{array}{c}46.25 \\
(\mathbf{4 2 . 8 4})^{*}\end{array}$ & $\begin{array}{c}48.46 \\
(\mathbf{4 4 . 1 2}) *\end{array}$ & $\begin{array}{c}53.17 \\
(\mathbf{4 6 . 8 2}) *\end{array}$ & 49.29 \\
\hline $\mathrm{T}_{3}$ & Metarhizium anisopliae & 3.1 & $\begin{array}{c}45.07 \\
(\mathbf{4 2 . 1 7})^{*}\end{array}$ & $\begin{array}{c}47.05 \\
(\mathbf{4 3 . 3 0})^{*}\end{array}$ & $\begin{array}{c}51.50 \\
(\mathbf{4 5 . 8 6})^{*}\end{array}$ & 47.87 \\
\hline $\mathrm{T}_{4}$ & Spinosad & 1.5 & $\begin{array}{c}73.75 \\
(\mathbf{5 9 . 1 8})^{*}\end{array}$ & $\begin{array}{c}77.07 \\
(\mathbf{6 1 . 3 9})^{*}\end{array}$ & $\begin{array}{c}82.17 \\
(\mathbf{6 5 . 0 5})^{*}\end{array}$ & 77.66 \\
\hline $\mathrm{T}_{5}$ & Quinalphos & 1.75 & $\begin{array}{c}65.65 \\
(\mathbf{5 4 . 1 2})^{*}\end{array}$ & $\begin{array}{c}66.92 \\
(\mathbf{5 4 . 8 9})\end{array}$ & $\begin{array}{c}73.72 \\
(\mathbf{5 9 . 1 7})\end{array}$ & 68.76 \\
\hline $\mathrm{T}_{6}$ & HaNPV & 2.05 & $\begin{array}{c}58.55 \\
(\mathbf{4 9 . 9 2 )}\end{array}$ & $\begin{array}{c}62.45 \\
(\mathbf{5 2 . 2 1})^{*}\end{array}$ & $\begin{array}{c}67.55 \\
(\mathbf{5 5 . 2 7})^{*}\end{array}$ & 62.85 \\
\hline $\mathrm{T}_{0}$ & $\begin{array}{l}\text { Untreated control } \\
\text { Water spray }\end{array}$ & 5.9 & $\begin{array}{c}0.0 \\
(\mathbf{0 . 5 4})^{*}\end{array}$ & $\begin{array}{c}0.0 \\
(\mathbf{0 . 5 4})^{*}\end{array}$ & $\begin{array}{c}0.0 \\
(\mathbf{0 . 5 4})^{*}\end{array}$ & \\
\hline & Over all Mean & & 49.60 & 52.09 & 56.04 & \\
\hline & F- test & & $\mathrm{S}$ & $\mathrm{S}$ & $\mathrm{S}$ & \\
\hline & S. Ed. ( $( \pm)$ & & 0.66 & 0.83 & 1.04 & \\
\hline & C. D. $(P=0.05)$ & & 1.39 & 1.74 & 2.20 & 3.20 \\
\hline
\end{tabular}

Table.4 Comparative efficacy of certain bio-pesticides against tomato fruit borer [Helicoverpa armigera (Hubner)] during Rabi season in tomato. ( Mean of 1st and 2nd spray) 2014-15

\begin{tabular}{|c|c|c|c|c|}
\hline & \multirow[b]{2}{*}{ Treatments } & \multicolumn{3}{|c|}{ \%reduction in Helicoverpa armigera } \\
\hline & & I $^{\text {st }}$ spray & II $^{\text {nd }}$ spray & Overall mean \\
\hline \multirow[t]{2}{*}{$\mathrm{T}_{1}$} & Verticillium lecanii & 53.54 & 61.63 & 57.58 \\
\hline & & $(47.04)^{*}$ & $(51.73)^{*}$ & $(49.37)^{*}$ \\
\hline \multirow[t]{2}{*}{$\mathrm{T}_{2}$} & Beauveria bassiana & 44.92 & 49.29 & 47.10 \\
\hline & & $(42.01)^{*}$ & $(44.59) *$ & $(43.33) *$ \\
\hline \multirow[t]{2}{*}{$\mathrm{T}_{3}$} & Metarhizium anisopliae & 41.05 & 47.87 & 44.46 \\
\hline & & $(39.82) *$ & $(43.77) *$ & $(41.81) *$ \\
\hline \multirow[t]{2}{*}{$\mathrm{T}_{4}$} & Spinosad & 72.28 & 77.66 & 74.97 \\
\hline & & $(58.23) *$ & $(61.86)^{*}$ & $(60.00) *$ \\
\hline \multirow[t]{2}{*}{$\mathrm{T}_{5}$} & Quinalphos & 63.86 & 68.76 & 66.31 \\
\hline & & $(53.05)^{*}$ & $(56.05)^{*}$ & $(54.52) *$ \\
\hline \multirow[t]{2}{*}{$\mathrm{T}_{6}$} & HaNPV & 56.63 & 62.85 & 59.74 \\
\hline & & $(48.81)^{*}$ & $(52.46)^{*}$ & $(50.62) *$ \\
\hline \multirow[t]{5}{*}{$\mathrm{T}_{0}$} & Untreated control & 0.0 & 0.0 & 0.0 \\
\hline & Water spray & $(0.54)^{*}$ & $(0.54)^{*}$ & $(0.54)^{*}$ \\
\hline & F- test & $\mathrm{S}$ & $\mathrm{S}$ & $S$ \\
\hline & S. Ed. $( \pm)$ & 1.82 & 1.2 & 0.90 \\
\hline & C. D. $(P=0.05)$ & 4.60 & 3.02 & 2.29 \\
\hline
\end{tabular}


Table.5 Economic of treated tomato crop of cultivation/ha

\begin{tabular}{|c|c|c|c|c|}
\hline S.n. & Particular & Requirement & Rate/unit Rs. & Cost \\
\hline (A) & Land preparation & & & \\
\hline I. & Ploughing & 3 hours & 500 Rs/hours & 1500 \\
\hline II. & Harrow & 3 hours & $500 \mathrm{Rs} /$ hours & 1500 \\
\hline III. & Layout of field \& showing & 15 labours & $150 \mathrm{Rs} / \mathrm{labour}$ & 2250 \\
\hline (B) & Manures and fertilizer & & & \\
\hline I. & Urea & $260 \mathrm{~kg}$ & $12 \mathrm{Rs} . / \mathrm{Kg}$ & 3120 \\
\hline II. & SSP & $150 \mathrm{~kg}$ & 7.8 Rs./Kg & 1170 \\
\hline III. & MOP & $90 \mathrm{~kg}$ & $19 \mathrm{Rs} . / \mathrm{Kg}$ & 1710 \\
\hline IV. & Labour & 2 labours & 150 & 300 \\
\hline (C) & Seed sowing & $400-500 \mathrm{gm} / \mathrm{ha}$. & 800 Rs./Kg & 400 \\
\hline I. & Seed material & 3 labours & & \\
\hline II. & Weeding & & 150Rs/labour & 450 \\
\hline (D) & Irrigation & & & \\
\hline I. & First time & 4 hours & 100 Rs./hours & 400 \\
\hline II. & Second time & 4 hours & 100 Rs./hours & 400 \\
\hline III. & Labour & 2 Labours & 100 Rs/labour & 200 \\
\hline (F) & Harvesting & 15 labours & 150 Rs/labour & 2250 \\
\hline I. & Labour & 6 Labour & 150 Rs/hours & 900 \\
\hline II. & Transport charge & 1 Truck & 5000 & 5000 \\
\hline (G) & Total cost of cultivation & & & 21550 \\
\hline
\end{tabular}

Table.6 Cost of Insecticides/ha

\begin{tabular}{|c|c|c|c|c|c|c|}
\hline Treatment & $\begin{array}{l}\text { Use of chemical } \\
\text { (2TimeSpray) }\end{array}$ & $\begin{array}{c}\text { Cost of } \\
\text { Insecticides }\end{array}$ & $\begin{array}{c}\text { Total Cost of } \\
\text { Insecticides } \\
\text { (Rs.) }\end{array}$ & $\begin{array}{l}\text { Use of } 2 \\
\text { labours } \\
\text { (Rs.) }\end{array}$ & $\begin{array}{c}\text { Total labour } \\
\text { cost (Rs.) }\end{array}$ & $\begin{array}{c}\text { Total cost of } \\
\text { Treatment }\end{array}$ \\
\hline \multicolumn{7}{|l|}{ Control } \\
\hline Verticilliumlecanii & $5 \mathrm{~kg} / \mathrm{ha}$ & $200 \mathrm{Rs} / \mathrm{kg}$ & 1000 & 150 & 600 & 1600 \\
\hline Beauveriabassiana & $5 \mathrm{~kg} . / \mathrm{ha}$ & $180 \mathrm{Rs} / \mathrm{kg}$ & 900 & 150 & 600 & 1500 \\
\hline $\begin{array}{c}\text { Metarhiziumanisoplia } \\
e\end{array}$ & $5 \mathrm{~kg} / \mathrm{ha}$ & 220Rs $/ \mathrm{kg}$. & 1100 & 150 & 600 & 1700 \\
\hline Spinosad & $900 \mathrm{ml} / \mathrm{ha}$ & $13000 \mathrm{Rs} / \mathrm{lit}$. & 9750 & 150 & 600 & 10350 \\
\hline Quinalphos & 2 lit/ha & 400Rs / lit. & 800 & 150 & 600 & 1400 \\
\hline HaNPV & $500 \mathrm{LE} / \mathrm{ha}$ & $200 \mathrm{Rs} / \mathrm{LE}$ & 800 & 150 & 600 & 1400 \\
\hline
\end{tabular}


Table.7 Economics of treatment

\begin{tabular}{ccccccccc}
\hline Treatment & $\begin{array}{l}\text { Yield } \\
\text { of q/ha }\end{array}$ & $\begin{array}{l}\text { Cost } \\
\text { yield } \\
\text { (Rs/q) }\end{array}$ & $\begin{array}{l}\text { Total cost } \\
\text { yield (Rs.) }\end{array}$ & $\begin{array}{l}\text { Common } \\
\text { cost (Rs.) })\end{array}$ & $\begin{array}{l}\text { Treatment } \\
\text { cost (Rs.) }\end{array}$ & $\begin{array}{l}\text { Total } \\
\text { cost (Rs.) }\end{array}$ & $\begin{array}{l}\text { C:B } \\
\text { ratio }\end{array}$ \\
\hline $\begin{array}{c}\text { Control } \\
\text { Verticillium } \\
\text { lecanii }\end{array}$ & 110 & 2000 & 220000 & 21550 & -- & 21550 & $1: 10.20$ \\
$\begin{array}{c}\text { Beauveria } \\
\text { bassiana }\end{array}$ & 165 & 2000 & 330000 & 21550 & 1600 & 23150 & $1: 14.25$ \\
$\begin{array}{c}\text { Metarhizium } \\
\text { anisopliae }\end{array}$ & 158 & 2000 & 316000 & 21550 & 1500 & 23050 & $1: 13.70$ \\
Spinosad & 200 & 2000 & 400000 & 21550 & 10350 & 31900 & $1: 12.53$ \\
Quinalphos & 180 & 2000 & 360000 & 21550 & 1400 & 22950 & $1: 15.68$ \\
HaNPV & 172 & 2000 & 344000 & 21550 & 1400 & 22950 & $1: 14.98$ \\
\hline
\end{tabular}

Treatments $T_{3}, T_{2}$ and $T_{1}, T_{6}$ were par with each other. $7^{\text {th }}$ day after $1^{\text {st }}$ spraying. The

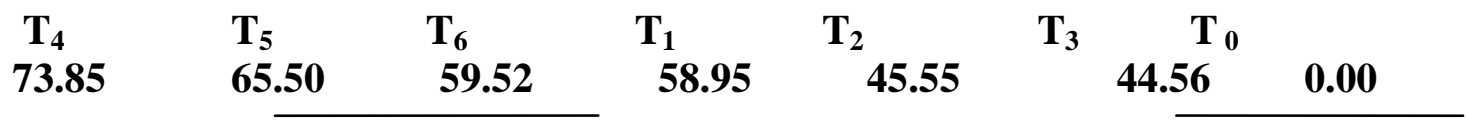

Some treatments were non significantly and some significantly superior over control. The data on population reduction percent of fruit borer (Helicoverpa armigera) on $7^{\text {th }}$ day after spraying showed that all the treatment were significantly superior over control Maximum reduction was observed with spinosad (73.85\%), followed by quinalphos $(65.50 \%)$, HaNPV (59.52\%), Beauveria bassiana

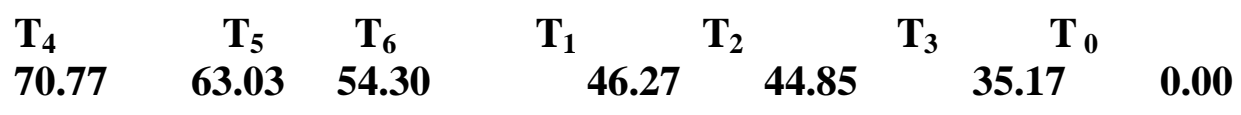

Treatments were significantly superior over Control. The data on population reduction percent reduction of Helicoverpa armigera given below. descending order of different treatment is given below.

(58.95\%), Verticillium lecanii (45.55\%), and Metarhizium anisopliae (44.46\%), were most effective treatment. Treatments $T_{3}, T_{2}$ and $T_{1}$, $\mathrm{T}_{6}$ were par with each other.

$14^{\text {th }}$ day after $1^{\text {st }}$ spraying

The descending order of different treatment is

over control on $14^{\text {th }}$ day after spraying revealed that the treatment were superior to control. The data on population reduction 
percent of fruit borer (Helicoverpa armigera) on $14^{\mathrm{en}}$ day after spraying showed that all the treatment were significantly superior over control Maximum reduction was observed with spinosad (70.77\%), followed by quinalphos (63.03\%), HaNPV (54.30\%), Beauveria bassiana (46.27\%), Verticillium lecanii (44.85\%), and Metarhizium

$\begin{array}{lcccc}\mathrm{T}_{4} & \mathrm{~T}_{5} & \mathrm{~T}_{6} & \mathrm{~T}_{1} & \mathrm{~T}_{2} \\ \mathbf{7 2 . 7 8} & \mathbf{6 3 . 8 6} & \mathbf{5 6 . 6 3} & \mathbf{5 3 . 5 4} & \mathbf{4 4 . 9}\end{array}$

Treatments were significantly superior over Control. The data on population reduction percent reduction of (Helicoverpa armigera) over control on $14^{\text {th }}$ day after spraying revealed that the treatment were superior to control. The data on population reduction percent of fruit borer (Helicoverpa armigera) on $1^{\text {st }}$ spraying mean showed that all the treatment were significantly superior over control Maximum reduction was observed with spinosad (72.28\%), followed by quinalphos (63.86\%), HaNPV (56.63\%), Beauveria bassiana (53.54\%), Verticillium lecanii (44.92\%), and Metarhizium anisopliae $(41.05 \%)$, were most effective treatment. each other. Siumilar finding was observed by Gandhi et al., (2013) evaluate the bio efficacy of spinosad $45 \mathrm{SC}(0.1 \mathrm{ml} / \mathrm{l})$, cypermethrin 10EC $(0.5 \mathrm{ml} / \mathrm{l})$, novaluran 10EC $(1 \mathrm{ml} / \mathrm{l})$, azardirachtin 5\% and Bacillus thringiensis (1 $\mathrm{ml} / \mathrm{l}$ ) insecticides against ear head caterpillar Helicoverpa armigera in sorghum, spinosad $45 \mathrm{SC}(0.1 \mathrm{ml} / 1)$, novaluran $10 \mathrm{EC}(1 \mathrm{ml} / \mathrm{l})$ and azardirachtin 5\% emerged as superior by recording72.0, 66.0 and $63.0 \%$ population reduction. Kale et al., (2008) reported that the Treatments $T_{3}, T_{2}$ and $T_{1}, T_{6}$ were par with

anisopliae (35.17\%), were most effective treatment. Treatments $\mathrm{T}_{2}$ and $\mathrm{T}_{1}$ were par with each other.

Mean of $I^{\text {st }}$ spray. $\left(3^{\text {rd }}, 7^{\text {th }}\right.$, and $\left.14^{\text {th }} \mathrm{DAS}\right)$

The descending order of different treatment is given below.

$$
\mathbf{T}_{3} \quad \mathbf{T}_{0}
$$

$\begin{array}{ll}41.05 & 0.00\end{array}$

treatments, spinosad $(0.01 \%)$ was most efficacious and recorded the lowest larval population and highest grain yield. Other effective microbial insecticides were HaNPV (250 LE ha-1) and Bt (750 ml ha-1), which stood only next to endosulfan $(0.06 \%)$. Metarhizium anisopliae (2.5 kg ha-1) and Beauveria bassiana (2.5 kg ha-1) were less efficacious, but performed better than their combinations with either HaNPV or Bt and combination of HaNPV with Bt at reduced doses

\section{Second spray}

\section{Reduction of fruit borer second (spray)}

The data on per cent reduction of fruit borer before spraying revealed that the results were statistically non significant.

\section{$3^{\text {rd }}$ day after $\mathrm{II}^{\text {nd }}$ spraying}

The descending order of different treatment is given below.

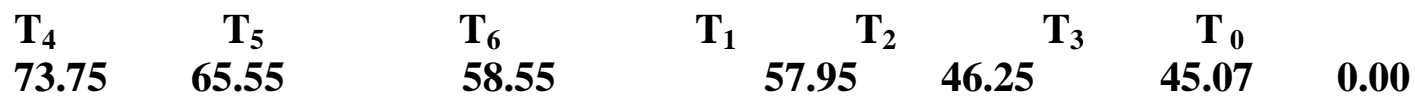

Some treatments were non significantly and some significantly superior over control. The data on population reduction percent of fruit borer (Helicoverpa armigera) on $3^{\text {rd }}$ day after spraying showed that all the treatment were significantly superior over control Maximum 
reduction was observed with spinosad (73.75\%), followed by quinalphos $(65.65 \%)$, HaNPV (58.55\%), Beauveria bassiana (57.95\%), Verticillium lecanii (46.25\%), and Metarhizium anisopliae (45.07\%), were most effective treatment. Treatments $\mathrm{T}_{3}, \mathrm{~T}_{2}$ and $\mathrm{T}_{1}$,

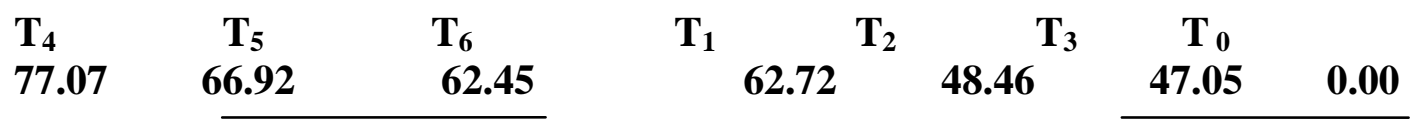

Some treatments were non significantly and some significantly superior over control. The data on population reduction percent of fruit borer (Helicoverpa armigera) on $7^{\text {th }}$ day after spraying showed that all the treatment were significantly superior over control Maximum reduction was observed with spinosad (77.07\%), followed by quinalphos $(66.92 \%)$, HaNPV (62.45\%), Beauveria bassiana

\section{$\begin{array}{lrlrl}\mathbf{T}_{4} & \mathrm{~T}_{5} & \mathrm{~T}_{6} & \mathrm{~T}_{1} & \\ \mathbf{8 2 . 1 7} & \mathbf{7 3 . 7 2} & \mathbf{6 7 . 5 5} & & \mathbf{6 4 . 2 2}\end{array}$}

Treatments were significantly superior over Control. The data on population reduction percent reduction of (Helicoverpa armigera) over control on $14^{\text {th }}$ day after spraying revealed that the treatment were superior to control. The data on population reduction percent of fruit borer (Helicoverpa armigera) on $14^{\text {en }}$ day after spraying showed that all the treatment were significantly superior over control Maximum reduction was observed with spinosad $(82.17 \%)$, followed by

\begin{tabular}{lcccccc}
$\mathbf{T}_{4}$ & $\mathrm{~T}_{5}$ & $\mathrm{~T}_{6}$ & $\mathrm{~T}_{1}$ & $\mathrm{~T}_{2}$ & $\mathrm{~T}_{3}$ & $\mathbf{T}_{\mathbf{0}}$ \\
$\mathbf{7 7 . 6 6}$ & $\mathbf{6 8 . 7 6}$ & $\mathbf{6 2 . 8 5}$ & $\mathbf{6 1 . 6 3}$ & $\mathbf{4 9 . 2 9}$ & $\mathbf{4 7 . 8 7}$ & $\mathbf{0 . 0 0}$ \\
\cline { 5 - 6 }
\end{tabular}

Treatments were significantly superior over Control. The data (Table 3) on population reduction percent reduction of Helicoverpa armigera over control on $14^{\text {th }}$ day after spraying revealed that the treatment were superior to control.

The data on population reduction percent of fruit borer Helicoverpa armigera on $2^{\text {nd }}$
$\mathrm{T}_{6}$ were par with each other.

$7^{\text {th }}$ day after II $^{\text {nd }}$ spraying

The descending order of different treatment is given below.

(62.72\%) Verticillium lecanii (48.46\%), and Metarhizium anisopliae (47.05\%), were most effective treatment. Treatments $\mathrm{T}_{3}, \mathrm{~T}_{2}$ and $\mathrm{T}_{1}$, $\mathrm{T}_{6}$ were par with each other.

\section{$14^{\text {th }}$ day after II $^{\text {nd }}$ spraying}

The descending order of different treatment is given below.

$\begin{array}{lllll}\mathbf{T}_{2} & & \mathbf{T}_{3} & \mathbf{T}_{\mathbf{0}} & \\ & \mathbf{5 3 . 1 7} & & \mathbf{5 1 . 5 0} & \mathbf{0 . 0 0}\end{array}$

quinalphos (73.72\%), HaNPV (67.55\%), Beauveria bassiana (64.22\%) Verticillium lecanii (53.17\%), and Metarhizium anisopliae $(51.50 \%)$, were most effective treatment. Treatments $T_{3}$ and $T_{2}$ were par with each other.

\section{Mean of $\mathrm{II}^{\text {st }}$ spray. $\left(3^{\text {rd }}, 7^{\text {th }}\right.$, and $14^{\text {th }}$ DAS $)$}

The descending order of different treatment is given below.

spraying mean showed that all the treatment were significantly superior over control maximum reduction was observed with spinosad (77.66\%), followed by quinalphos (68.76\%), HaNPV (62.85\%), Beauveria bassiana (61.63\%) Verticillium lecanii (49.29\%), and Metarhizium anisopliae $(47.87 \%)$ were most effective treatment. 
Treatments $T_{3}, T_{2}$ and $T_{1}, T_{6}$ were par with each other.

Randhawa et al., (2009) reported that five insecticides, i.e. endosulfan $35 \mathrm{EC}$ at $1250 \mathrm{ml}$ endosulfan $35 \mathrm{EC}$ at $2500 \mathrm{ml}$, spinosad $48 \mathrm{SC}$ at $150 \mathrm{ml}$, indoxacarb $15 \mathrm{EC}$ at $500 \mathrm{ml}$, cypermethrin $25 \mathrm{EC}$ at $200 \mathrm{ml}$, chlorpyriphos $20 \mathrm{EC}$ at $2500 \mathrm{ml}$ per hectare, along with untreated control, were evaluated against gram caterpillar (Helicoverpa armigera) on seed crop of berseem. Spinosad 48 SC was found to be the most effective insecticide for the control of $H$. armigera, followed closely

\section{$\begin{array}{lllll}\mathrm{T}_{4} & \mathrm{~T}_{5} & \mathrm{~T}_{6} & \mathrm{~T}_{1} & \mathrm{~T}_{2} \\ 74 & 63.86 & 56.63 & \end{array}$ 72.78 63.86 \\ 53.54}

Treatments were significantly superior over Control. The data on population reduction percent reduction of (Helicoverpa armigera) over control on $14^{\text {th }}$ day after spraying revealed that the treatment were superior to control. The data on population reduction percent of fruit borer (Helicoverpa armigera) on $1^{\text {st }}$ spraying mean showed that all the treatment were significantly superior over control Maximum reduction was observed with spinosad (72.28\%), followed by quinalphos (63.86\%), HaNPV (56.63\%),

\begin{tabular}{|c|c|c|c|c|c|}
\hline $\begin{array}{c}T_{4} \\
77.66\end{array}$ & $\begin{array}{c}T_{5} \\
68.76\end{array}$ & $\begin{array}{l}T_{6} \\
62.85\end{array}$ & $\begin{array}{l}T_{1} \\
61.63\end{array}$ & $\begin{array}{l}T_{2} \\
49.29\end{array}$ & $\mathrm{~T}_{3}{ }_{47.87}^{\mathrm{T}_{0}}$ \\
\hline
\end{tabular}

Treatments were significantly superior over Control. The data on population reduction percent reduction of (Helicoverpa armigera) over control on $14^{\text {th }}$ day after spraying revealed that the treatment were superior to control. The data on population reduction percent of fruit borer (Helicoverpa armigera) on $2^{\text {nd }}$ spraying mean showed that all the treatment were significantly superior over control Maximum reduction was observed with spinosad (77.66\%), followed by by indoxacarb15 EC. Phukon et al., (2014) revealed the reduction in fruit damage was up to 92.20 per cent in cypermethrin treated plot followed by 91.12 per cent, 88.74 per cent and 87.01 per cent in the plots treated with Neem oil, B. Bassiana and M. Anisopliae, respectively due to $H$. armigera larvae over control. The highest increase in yield over control was noticed in cypermethrin treated plots $(62.85 \%)$ followed by neem oil treated plots (41.83\%).

The descending order of different treatment is given below.

$44.92 \quad \stackrel{T_{3}}{41.05} \quad T_{0} 0.00$

Beauveria bassiana (53.54\%) Verticillium lecanii (44.92\%), and Metarhizium anisopliae $(41.05 \%)$, were most effective treatment. Treatments $T_{3}, T_{2}$ and $T_{1}, T_{6}$ were par with each other.

Mean of $\mathrm{II}^{\text {st }}$ spray. $\left(3^{\text {rd }}, 7^{\text {th }}\right.$, and $\left.14^{\text {th }} \mathrm{DAS}\right)$

The descending order of different treatment is given below.

quinalphos (68.76\%), HaNPV (62.85\%), Beauveria bassiana (61.63\%) Verticillium lecanii (49.29\%), and Metarhizium anisopliae (47.87\%), were most effective treatment. Treatments $T_{3}, T_{2}$ and $T_{1}, T_{6}$ were par with each other.

\section{Over all mean of first and second spray}

The descending order of different treatment is given below. 


$\begin{array}{ccccccc}\mathbf{T}_{4} & \mathrm{~T}_{5} & \mathrm{~T}_{6} & \mathrm{~T}_{1} & \mathbf{T}_{2} & \mathbf{T}_{3} & \mathbf{T}_{\mathbf{0}} \\ \mathbf{7 4 . 9 7} & \mathbf{6 6 . 3 1} & \mathbf{5 9 . 7 4} & \mathbf{5 7 . 5 8} & \mathbf{4 7 . 1 0} & \mathbf{4 4 . 4 6} & \mathbf{0 . 0 0}\end{array}$

Treatments were significantly superior over Control. The data (Table 4) population reduction percent reduction of (Helicoverpa armigera) over control on $14^{\text {th }}$ day after spraying revealed that the treatment were superior to control.

The data on population reduction percent of fruit borer (Helicoverpa armigera) on 1st and $2^{\text {nd }}$ spraying mean showed that all the treatment were significantly superior over control Maximum reduction was observed with spinosad (74.97\%), followed by quinalphos (66.31\%), HaNPV (59.74\%), Beauveria bassiana (57.58\%), Verticillium lecanii (47.10\%), and Metarhizium anisopliae $(44.46 \%)$, were most effective treatment. Treatments $T_{1}$ and $T_{6}$ were par with each other. Similar founding was observed by

Singh et al., (2009) reported that spinosad 45 SC proved to be the best treatment in reducing the incidence in shed fruiting bodies and damage on boll and loculi basis followed by novaluron @ 50 and $37.5 \mathrm{~g}$ a.i./ha. Although the incidence of American bollworm was less in spinosad but yield was comparatively higher in novaluron @ 50 and $37.5 \mathrm{~g}$ a.i/ha as against spinosad and chlorpyriphos. Lad et al., (2009) reported that Metarhizium anisopliae @ 1x108 conidia ha-1, Beaveria bassiana $1 \times 108$ conidia ha-1, Metarhizium anisopliae @1×106 conidia ha-1, Beaveria bassiana 1x106 conidia ha-1, were at par with each other, but significantly inferior to first three biological treatments. However, the microbials were significantly better than Neemark and NSE 5\%.

\section{Cost benefit ratio}

The tables 5,6 and 7 with respect to cost benefit ratio $(\mathrm{CB})$ as influenced by various treatments is presented in table 4.8 and which revealed that the higher amount of monetary return was obtained with quinalphos $25 \mathrm{EC}$ (1:15.68) followed by HaNPV 250LE/ha (1:14.98), Verticillium lecanii (1:14.25), Beauveria bassiana (1:13.70), Metarhizium anisopliae (1:12.90) spinosad $45 \mathrm{SC}$ (1:12.53), and Control (1:14.84). The similar finding was observed by the following researcher Naik et al., (2013) reported that among the insecticides and biopesticides evaluated against $H$. armigera, the insecticide quinalphos (0.05) recorded the least leaf damage of $9.55 \%$ and it was at par with chlorpyriphos (0.05) (11.22\%). Highest pod yield of $9.86 \mathrm{q} / \mathrm{ha}$ was recorded with chlorpyriphos (0.05) followed by quinalphos(0.05) $9.41 \mathrm{q} / \mathrm{ha}$. The maximum cost:benefit ratio of 1:6.12 was obtained in quinalphos (0.05). Tayde and Simon (2010) revealed that Spinosad 45 SC @ $0.01 \%$ was found most effective and showed $(09.84 \%)$ shoot infestation, per cent fruit infestation $(06.87 \%$ on number basis and $07.35 \%$ on weight basis) and increasing yield of brinjal fruit $(239.30 \mathrm{q} / \mathrm{ha})$. Whereas, carbaryl $50 \mathrm{WP}$ @ $0.2 \%$ and endosulfan 35 EC @ $0.05 \%$ were also found effective in reducing per cent infestation shoot and fruit infestation and increasing yield. Amongst neem products NSKE 5\% was found to be superior in terms of efficacy and yield. However, the increment cost benefit ratio (ICBR) showed that the application of quinalphos 25 EC @ 0.05\% was economically most viable treatment (1:67.86) followed by endosulfan 35 EC @ $0.05 \%$ (1:66.19).

\section{References}

Bhutani, D.K. 1977. Insects of vegetable: tomato. Pesticides, 11(1): 33-36

Choudary, B.R. 2002. Important of tomato 
vegetable crop. P. B. 121(4): 292-296.

Dhaka, S.S., Singh, G., Ali, N., Yadav, A. and Yadav, A. 2010. Field evaluation of insecticides and bio-pesticides against (Helicoverpa armigera) on tomato. Annals of Plant Protection Sci., 18(1): 13-16.

Gandhi, B. K., Shekharappa, and Balikai, R. A. 2013. Bio-efficacy of insecticides in management of Helicoverpa armigera (Hubner) in kharif sorghum. Annals of Plant Protection Sci., 21(1): 83-86.

Kale, S. N., Men, U. B. 2008. Efficacy of microbial insecticides and their combinations against Helicoverpa armigera (Hubner) on chickpea. J. Biol. Control, 22(1): 205-208.

Katyayan, A. 2010. Fundamentals of Agriculture, Kushal Publication and Distributors Varanasi, (2): 215-216.

Kaur, Sandeep, and Subash, Singh. 2013. Field efficacy of some systemic insecticides and microbial pesticides (modules) against aphid, aphis gossypii glover and fruit borer, Helicoverpa armigera (hubner) on tomato in Punjab. Agri. Sustainable., 1(1): 1-6.

Khanam, U.K.S., Hossian, M., Ahmed, N., Uddin, M.M. and Hossain, M.S. 2003. Varietal screening of tomato fruit borer, Helicoverpa armigera (Hub.) and associated tomato characters. Pakistan J. Biol. Sci., 6(4): 413-421.

Lad, S. K., Peshkar, L. N., Baviskar, S. S., Jadhav, R. S. 2009. Efficacy of microbials and bioagents for the management of Plutella xylostella (L.) on cauliflower. J. Soils and Crops, 19(1): 129-134.

Manjunath, T.M., Bhatnagar, V.S., Pawar, C.S. and Sithanathan. 1987. Economic importance of Heliothis armigera spp. In India and assessment of their natural enemies and host plants. Proceeding of the International Workshop on Biological on Heliothis, 11-15
November, 1985, New Delhi, India.

Naik, C. M., Chakravarthy, A. K., Naik, T. B. and Sasivihalli, P. B. 2013. Population dynamics and management of Helicoverpa armigera (Hubner) (Noctuidae:Lepidoptera) on groundnut in coastal Karnataka. Environ. Ecol., 31(1): 54-57.

Phukon, M., Sarma, I., Borgohain, R., Sarma, B. and Goswami, J. 2014. Efficacy of Metarhizium anisopliae, Beauveria bassiana and neem oil against tomato fruit borer, Helicoverpa armigera under field condition. Asian J. Bio Sci., 9(2): 151-155.

Randhawa, H. S., Aulakh, S. S., Bhagat, I. Chhina, J. S. 2009. Efficacy of different insecticides against Helicoverpa armigera (Hubner) (Lepidoptera: Noctuidae) on seed crop of berseem in Punjab. Legume Res., 32(2): 145-148.

Ravi, M., Santharam, G. and Sathiah, N. 2008. Eco friendly management of tomato fruit borer, Helicoverpa armigera (Hubner). J. Biopesticides, 1(2): 134-137.

Selvanarayanan, V. and Narayanasamy, P. 2006. Factors of resistance in tomato accessions against the fruit worm, Helicoverpa armigera (Hubner). Crop Protection, 25(10): 1075-1079.

Shinde, S.T., Shetgar S.S. and Badgujar A.G. 2011. Bio-efficacy of different insecticides against major pest of okra. J. Entomol. Res., 35(2): 133-137.

Singh Ravinder, Dhawan, A. K. and Shera, P. S. 2009. Field efficacy of novaluron (Rimon 10 EC) against bollworms on cotton. J. Insect Sci., 22(4): 343-350.

Singh, H. and Singh, G. 1975. Biological studies on Heliothis armigera (Hubner) in the Punjab. Indian J. Entomol., 37(2): 156-164.

Sivaprakasam, N. 1996. Influence of trichomes on theresistance to fruit borer, Helicoverpa armigera in tomato. 
Madras Agri. J., 83(5): 306-307.

Biosci., 3(2): 208-209.

Sreekanth, and Seshamahalakshmi. 2010.

Studies on relative toxicity of bio pesticides to Helicoverpa armigera (Hubner) and Maruca vitrata (Geyer) on pigeonpea (Cajanus cajan L.) J. Biopesticide., 5(2): 191-195.

Tayde, A.R. and Sobita, Simon. 2010. Efficacy of spinosad and neem products against shoot and fruit borer, Leucinodes orbonalis (Guen.) of brinjal, (Solanum melongena L.). Trends in

Tripathi, R. and Singh, N. P. 2005. Field efficacy of bio pesticide and insecticide against Helicoverpa armigera on tomato crop. Shashpa, 12(1): 65-66.

Yadav, D.N. 1980. Studies on the natural enemies of Heliothis armigera (Hubner) and its biological control using an egg parasite, Trichogramma australicum Giranit. (Hymenoptera, Trichogramatidae). G.A.U. Res. J., 6(1): 62-63.

\section{How to cite this article:}

Renu Choudhary, Ashwani Kumar, G.C. Jat, Vikram and Deshwal, H.L. 2017. Comparative Efficacy of Certain Bio-Pesticides against Tomato Fruit Borer, Helicoverpa armigera (Hub.). Int.J.Curr.Microbiol.App.Sci. 6(8): 1068-1081. doi: https://doi.org/10.20546/ijcmas.2017.608.133 